\title{
XMM-Newton and INTEGRAL observations of new absorbed supergiant high-mass X-ray binaries
}

\author{
R. Walter ${ }^{1,2}$, J. Zurita Heras ${ }^{1,2}$, L. Bassani ${ }^{3}$, A. Bazzano ${ }^{3}$, A. Bodaghee ${ }^{1,2}$, A. Dean ${ }^{4}$, \\ P. Dubath ${ }^{1,2}$, A. N. Parmar ${ }^{6}$, M. Renaud ${ }^{7}$, and P. Ubertini ${ }^{3}$ \\ 1 INTEGRAL Science Data Centre, Chemin d'Écogia 16, 1290 Versoix, Switzerland \\ e-mail: Roland.Walter@obs.unige.ch \\ 2 Observatoire de Genève, Chemin des Maillettes 51, 1290 Sauverny, Switzerland \\ 3 IASF/INAF, via Godetti 101, 40129 Bologna, Italy \\ 4 IASF/INAF, via Fosso del Cavaliere 100, 00133 Rome, Italy \\ 5 School of Physics and Astronomy, University of Southampton, Highfield, Southampton, SO 17 1BJ, UK \\ ${ }^{6}$ Astrophysics Missions Division, Research and Scientific Support Department of ESA, ESTEC, Postbus 299, 2200 AG Noordwijk, \\ The Netherlands \\ 7 CEA Saclay, DSM/DAPNIA/SAp, 91191 Gif-sur-Yvette Cedex, France
}

Received 29 June 2005 / Accepted 17 March 2006

\section{ABSTRACT}

\begin{abstract}
Context. During the first year in operation, INTEGRAL, the European Space Agency's $\gamma$-ray observatory, has detected more than 28 new bright sources in the galactic plane which emit the bulk of their emission above $10 \mathrm{keV}$.

Aims. To understand the nature of those sources we have obtained follow-up observations in the X-ray band with XMM-Newton. Methods. We derive accurate X-ray positions, propose infrared counterparts and study the source high energy long and short term variability and spectra.

Results. $70 \%$ of the sources are strongly absorbed $\left(N_{\mathrm{H}} \geq 10^{23}\right.$ atom $\left.\mathrm{cm}^{-2}\right)$. More than half of these absorbed sources show pulsations with periods ranging from 139 to 1300 s, i.e., they are slow X-ray pulsars. The candidate infrared counterparts are not as strongly absorbed demonstrating that part of the absorbing matter is local to the sources.

Conclusions. Many of these new sources are supergiant high-mass X-ray binaries (HMXB) in which the stellar wind of the companion star is accreted onto the compact object. The large local absorption in these new sources can be understood if the compact objects are buried deep in their stellar winds. These new objects represent half of the population of supergiant HMXB.
\end{abstract}

Key words. gamma-rays: observations - X-rays: binaries - stars: pulsars: general - stars: early type

\section{Introduction}

High-mass X-ray binaries (HMXBs) consist of a compact object orbiting a massive star. The X-ray emission is due to the accretion of matter from the early-type mass-losing star onto the compact companion, which is generally a neutron star. HMXBs can be divided into two groups (van Paradijs 1983) depending on the nature of the massive star.

Most of the 130 HMXBs cataloged by Liu et al. (2000) belong to the first group and contain a Be primary star in a relatively wide orbit of moderate eccentricity. The compact companions spend most of the time far away from the disk surrounding the Be star (van Paradijs 1995; Apparao 1994). X-ray outbursts occur primarily during the perigee passage of the compact object which accretes from a low-velocity and high-density wind from the Be star. These systems exhibit hard X-ray spectra and tend to be transient X-ray emitters.

In the second group, the compact object orbits inside the wind of an OB supergiant star. The X-ray emission is either powered by direct accretion of the stellar wind or by Rochelobe overflow via an accretion disk. The X-ray emission from these systems is generally persistent. They can provide insights into the masses of neutron stars, the structure of stellar winds and binary evolution. These systems may ultimately evolve into highly interesting neutron star - black hole binaries. However, supergiant X-ray binaries are rare, with only 10 such systems known in the Galaxy prior to INTEGRAL (e.g., Liu et al. 2000) and so the discovery of new examples is of great interest.

The INTEGRAL survey of the galactic plane and central regions have so far revealed the presence of $\sim 120$ sources in the energy range $20-100 \mathrm{keV}$. Sources are located with an accuracy better than $2^{\prime}$, depending on count rate, position in the field of view (FOV) and exposure. This excellent source location accuracy has allowed the majority of the detected sources to be identified with already known X-ray sources. However, 28 of the objects in the first INTEGRAL soft $\gamma$-ray galactic plane survey catalog (Bird et al. 2004) had no known counterparts and so their nature was unclear. The majority of those sources were detected in the galactic bulge and Norma arm tangent region (Walter et al. 2004).

XMM-Newton follow-up observations of the first of the newly-detected INTEGRAL source, IGR J16318-4848, revealed a spectrum with intense $\mathrm{Fe} \mathrm{K} \alpha$ and $\mathrm{K} \beta$ and $\mathrm{Ni} \mathrm{K} \alpha$ emission features as well as strong $\left(\gtrsim 10^{24}\right.$ atom $\left.\mathrm{cm}^{-2}\right)$ low-energy absorption which makes the source extremely difficult to observe at energies $\lesssim 4 \mathrm{keV}$ (Matt \& Guainazzi 2003; Walter et al. 2003b; Revnivtsev et al. 2003). The strong absorption and unusual spectral properties led to the suggestion that IGR J16318-4848 is 
the first representative of a group of highly absorbed galactic binaries.

Following the discovery of IGR J16318-4848, there have been a number of sensitive X-ray observations of some of the other newly-discovered INTEGRAL sources. These observations have revealed that several of the sources share similar spectral properties (Rodriguez et al. 2003; Patel et al. 2004).

Here, we present the results of INTEGRAL and XMM-Newton observations of 10 newly-discovered sources. These were selected among the new sources detected by INTEGRAL in the galactic plane during the first year of the mission. We also collected infrared photometry for the candidate counterparts from existing catalogues. We demonstrate that 8 out of the 10 sources are intrinsically absorbed and that 8 of them are persistent X-ray emitters. Many systems appear to have supergiant companions. Since the X-ray properties of the other unknown systems are similar, we propose that significant numbers of the unknown sources are likely to be OB supergiant systems - doubling the number of such systems known in the Galaxy.

\section{Observations and data analysis}

\subsection{INTEGRAL}

The INTEGRAL payload (Winkler et al. 2003) consists of two $\gamma$ ray instruments, one of which is optimized for $15 \mathrm{keV}$ to $10 \mathrm{MeV}$ high-resolution imaging (IBIS; Ubertini et al. 2003) and the other for $20 \mathrm{keV}$ to $8 \mathrm{MeV}$ high-resolution spectroscopy (SPI; Vedrenne et al. 2003). IBIS provides an angular resolution of $12^{\prime}$ full-width at half-maximum $(F W H M)$ and an energy resolution, $E / \Delta E$, of $\sim 12 F W H M$ at $100 \mathrm{keV}$. SPI provides an angular resolution of $2.7 F W H M$ and an $E / \Delta E$ of $\sim 500 F W H M$ at $1.3 \mathrm{MeV}$. The extremely broad energy range of IBIS is covered by two separate detector arrays, ISGRI (15-500 keV, Lebrun et al. 2003) and PICsIT (0.2-10 MeV, Labanti et al. 2003). The payload is completed by X-ray (JEM-X; 3-35 keV, Lund et al. 2003) and optical monitors (OMC; $V$-band, Mas-Hesse et al. 2003). The instruments are co-aligned and are operated simultaneously.

The 3 high-energy instruments use coded masks to provide imaging information. This means that photons from a source within the FOV are distributed over the detector area in a pattern determined by the position of the source in the FOV. Source positions and intensities are determined by matching the observed distribution of counts with those produced by the mask modulation.

Most of the new sources discovered by INTEGRAL could be detected with ISGRI in mosaics of sky images obtained in hundreds of short $(2000 \mathrm{~s})$ pointings scheduled during each of the source visibility periods (a source is visible during about 2 months every 6 months).

Table 1 lists the net ISGRI effective exposure time and the average source count rate obtained during the first year of the mission for the new sources for which high-resolution X-ray follow-up observations were performed.

The INTEGRAL data were processed using the Off-line Scientific Analysis (OSA) version 4.2 software provided by the INTEGRAL Science Data Centre (Courvoisier et al. 2003).

Extracting source spectra with OSA 4.2 in each pointing and averaging the results is a method affected by systematic errors that are large for sources of low significance. We therefore extracted source average spectra from mosaic images, which is a more reliable approach. Although source count rates extracted
Table 1. INTEGRAL ISGRI vignetting-corrected effective exposure time and average source count rate during the first year of the mission. $3 \sigma$ upper limits are provided when the source was not detected during that period.

\begin{tabular}{lcc}
\hline \hline Source & $\begin{array}{c}\text { Eff Exp } \\
{[\mathrm{ks}]}\end{array}$ & $\begin{array}{c}20-60 \mathrm{keV} \mathrm{count} \mathrm{rate} \\
{[\mathrm{ct} / \mathrm{s}]}\end{array}$ \\
\hline \multicolumn{2}{l}{ PERSISTENT SOURCES } \\
IGR J16318-4848 & 554 & $3.52 \pm 0.02$ \\
IGR J16320-4751 & 510 & $2.24 \pm 0.02$ \\
IGR J16393-4641 & 387 & $1.16 \pm 0.02$ \\
IGR J16418-4532 & 489 & $0.95 \pm 0.02$ \\
IGR J16479-4514 & 532 & $0.75 \pm 0.03$ \\
IGR J17252-3616 & 1248 & $1.48 \pm 0.02$ \\
IGR J17597-2201 & 288 & $1.40 \pm 0.03$ \\
IGR J18027-2016 & 276 & $0.85 \pm 0.07$ \\
TRANSIENT SOURCES & \\
IGR J16465-4507 & 504 & $<0.08$ \\
IGR J17544-2619 & 208 & $<0.12$ \\
\hline
\end{tabular}

from mosaic images are 5\% less than obtained using other spectral extraction methods (Lubinski et al. 2004), the source relative variability and spectral shape are not affected.

The ISGRI source positions and average spectra were derived with the mosaic_spec (version 1) software from a set of mosaic images generated in narrow energy bands from the data of the first two visibility periods (the first year of the mission). For IGR J16465-4507 and IGR J17544-2619, which are transient sources, the ISGRI spectra were derived from revolutions 232/233 (MJD 53 254.34-53 257.34) and 113 (MJD 52 898.35$52901.35)$ respectively.

To study the long term variability of the sources, we included Core Programme data from the second year of the mission. The lightcurves, with a binning of one spacecraft revolution (3 days), were extracted from mosaic images built for each revolution.

\subsection{XMM-Newton}

The XMM-Newton Observatory (Jansen et al. 2001) includes three $1500 \mathrm{~cm}^{2} \mathrm{X}$-ray telescopes each with a European Photon Imaging Camera (EPIC) at the focus. Two of the EPIC imaging spectrometers use MOS CCDs (Turner et al. 2001) and one uses pn CCDs (Strüder et al. 2001).

All sources listed in Table 2 were observed by the EPIC camera of XMM-Newton with the medium (thick in the case of IGR J16465-4507, thin in the case of IGR J16320-4751) optical blocking filter. The EPIC pn cameras were operated in largewindow mode. Pile-up is negligeable for all sources.

For this paper, only the EPIC data were considered. Source positions were derived using the SAS task edetect_chain from the EPIC MOS and pn data. Source spectra and lightcurves were derived from EPIC pn data (MOS data are consistent but of lower significance).

All data products were obtained using the Science Analysis Software (SAS) version 6.1.0. Only single and double pixel events were selected. Known hot, or flickering, pixels and electronic noise were rejected. Source events were selected in a circle centered on the source. Background events were obtained from source-free regions of identical size located in the same CCD and at the same distance from the read-out node. 
Table 2. XMM-Newton EPIC pn observation log. All EPIC observations used the medium filter (except IGR J16465-4507 and IGR J16320-4751 for which the thick and thin, filters were used respectively) and have been performed in large window mode. The third column gives the net time accumulated in the source spectrum. The last column $\left(B_{\text {cut }}\right)$ gives the threshold in detector count rate above $10 \mathrm{keV}$ used to remove periods with high background.

\begin{tabular}{|c|c|c|c|}
\hline Source & Start time & $\begin{array}{l}\text { Net Exp } \\
{[\mathrm{ks}]}\end{array}$ & $\begin{array}{r}B_{\text {cut }} \\
{[\mathrm{ct} / \mathrm{s}]}\end{array}$ \\
\hline \multicolumn{4}{|c|}{ PERSISTENT SOURCES } \\
\hline IGR J16318-4848 & $2003-02-10 \quad 16: 21$ & 21.1 & 60 \\
\hline IGR J16320-4751 & 2004-08-19 17:07 & 33.1 & \\
\hline IGR J16393-4641 & 2004-03-21 08:59 & 6.9 & 1 \\
\hline IGR J16418-4532 & 2004-08-19 06:30 & 10.5 & 2 \\
\hline IGR J16479-4514 & $2004-03-2122: 18$ & 5.8 & 60 \\
\hline IGR J17252-3616 & $2004-03-21 \quad 13: 23$ & 5.5 & 4 \\
\hline IGR J17597-2201 & 2004-03-21 22:18 & 2.4 & 30 \\
\hline IGR J18027-2016 & 2004-04-06 06:55 & 7.6 & 6 \\
\hline \multicolumn{4}{|c|}{ TRANSIENT SOURCES } \\
\hline IGR J16465-4507 & 2004-09-14 02:46 & 2.9 & 1 \\
\hline IGR J17544-2619 & 2003-09-11 19:25 & 4.9 & 1 \\
\hline
\end{tabular}

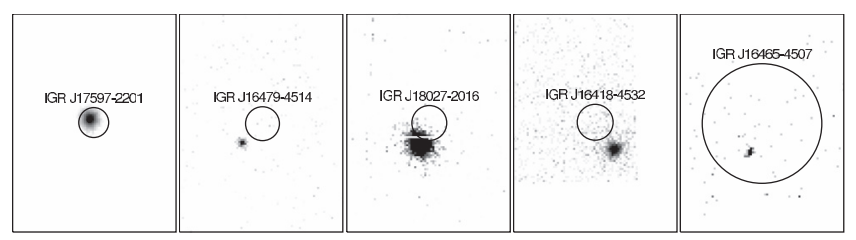

Fig. 1. EPIC counterpart images for 5 INTEGRAL sources for which that information is not presented elsewhere. North is left. The size of each image is $2.8 \times 3.7$ arcmin. The ISGRI $90 \%$ uncertainty regions (not including systematics of the reconstruction) are shown.

Periods with enhanced background (EPIC pn count rate above $10 \mathrm{keV}$ larger than the threshold listed in Table 2) were disregarded in the analysis.

\section{Results}

\subsection{Positions and counterparts}

The statistical accuracy on the determination of the position of point sources with ISGRI depends on the source significance in the mosaic image and varies from 2 arcmin to 10 arcsec in our sample. ISGRI source positions with $90 \%$ uncertainty radius (including a 10 arcsec systematic uncertainty on the instrument misalignment - Walter et al. 2003a) are listed in Table 3. ISGRI positions derived by the current software are affected by additional systematic errors related with the image reconstruction. These errors are proportional to the source significance and amount to a good fraction of the statistical uncertainties (Gros et al. 2003).

EPIC pn images with superimposed ISGRI error circles are presented in Fig. 1 if not already presented elsewhere (Bodaghee et al. 2005; Zurita Heras et al. 2005; Walter et al. 2003b; Rodriguez et al. 2003; González-Riestra et al. 2004). XMM-Newton detected an X-ray counterpart for all the INTEGRAL sources observed. The counterpart detected by EPIC are often on the border of the ISGRI error circles because of the systematic errors. The X-ray positions derived from

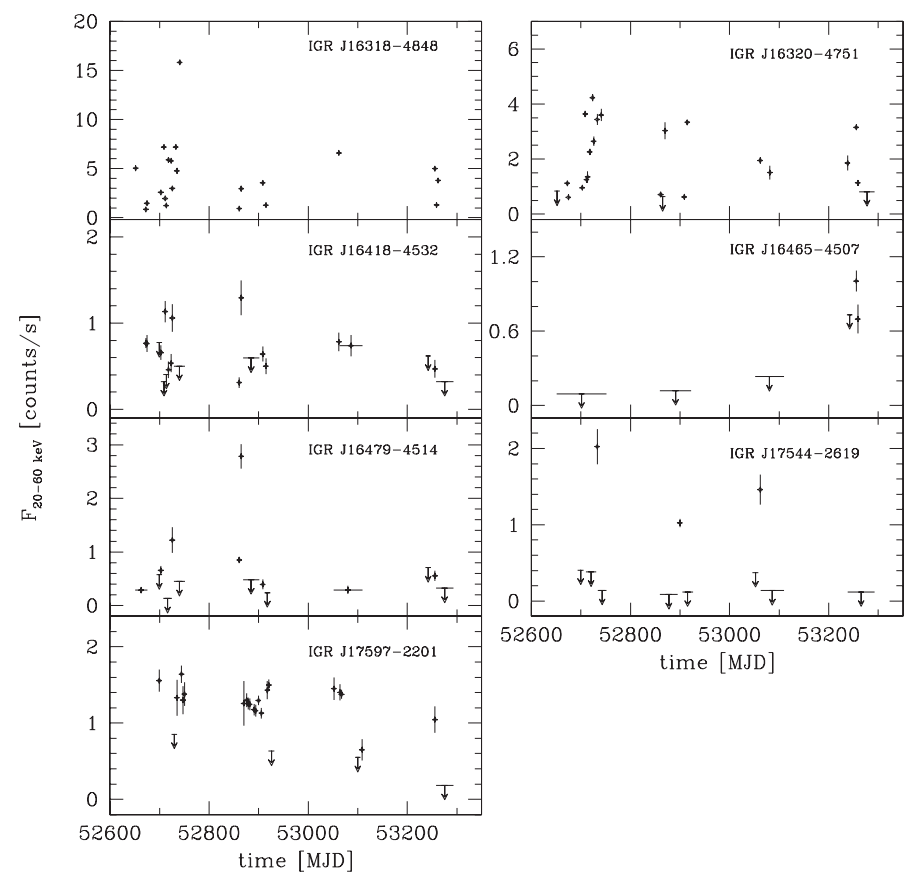

Fig. 2. INTEGRAL ISGRI long term source lightcurves (20-60 keV).

follow-up observations of our sample of sources are listed in Table 3.

We searched in several catalogues for candidate counterparts. None of the sources are detected in the radio. The only known optical/infrared candidate counterparts found within the $\mathrm{X}$-ray error circles are listed in Table 3 . The coordinates come from the 2MASS catalogue excepting for the second candidate counterpart of IGR J18027-2016 which was found in the Second Guide Star Catalogue. In most cases a single candidate counterpart was found. The closest 2MASS source to IGR J17597-2201 lies outside of the X-ray error circle and may not be the real stellar counterpart.

\subsection{Long term variability}

To study the long term variability of the sources we have constructed ISGRI 20-60 keV lightcurves with one time bin every one (or several when the source was not detected) 3 day spacecraft revolution. The count rate in each time bin were extracted from a mosaic of all pointings performed during the revolution(s). When the source was not detected in a time bin, a $3 \sigma$ upper limit was derived from the variance map. Only revolutions with an effective exposure time on source longer than $5000 \mathrm{~s}$ have been considered.

Figure 2 displays the long term lightcurve of all selected sources except IGR J16393-4641, IGR J17252-3616, IGR J18027-2016 for which similar information can be found in Bodaghee et al. (2005); Zurita Heras et al. (2005) and Hill et al. (2005).

The first 8 sources listed in Table 1 are significantly detected during each of the periods of visibility. These sources were persistent, at least on a time scale of 2 years, but vary by factor 5 to 20 at least. Weak detection of all these sources, excepting IGR J16418-4532, by previous mission (Murakami et al. 2003; Rodriguez et al. 2003; Sugizaki et al. 2001; Warwick et al. 1988; Augello et al. 2003; Lutovinov et al. 2005a) also support their persistent nature. 
Table 3. Consolidated position of the INTEGRAL sources with X-ray and candidate infrared counterparts. The $K$ magnitude and the angular separation, $\Delta r$, between the infrared counterpart and the X-ray source are also indicated.

\begin{tabular}{|c|c|c|c|c|c|}
\hline Source & ISGRI position (uncertainty ["]) & X-ray position (uncertainty ["] & 2MASS & $K$ & $\Delta r\left[{ }^{\prime \prime}\right]$ \\
\hline \multicolumn{6}{|c|}{ PERSISTENT SOURCES } \\
\hline IGR J16318-4848 & $163148-4849.0(13)$ & $163148.6-484900(4)^{1}$ & $16314831-4849005$ & 7.2 & 2.9 \\
\hline IGR J16320-4751 & $163202-4752.2(16)$ & $163201.9-475227(3)^{2}$ & $16320175-4752289$ & 10.9 & 1.9 \\
\hline IGR J16393-4641 & $163906-4642.1(26)$ & $163905.4-464212(4)^{3}$ & $16390535-4642137$ & 12.8 & 1.8 \\
\hline IGR J16418-4532 & $164146-4531.8(36)$ & $164151.0-453225$ (4) & $16415078-4532253$ & 11.5 & 2.4 \\
\hline IGR J16479-4514 & $164803-4512.8(34)$ & $164806.6-451208(4)$ & $16480656-4512068$ & 9.8 & 1.3 \\
\hline IGR J17252-3616 & $172510-3617.3(22)$ & $172511.3-361658(4)^{4}$ & $17251139-3616575$ & 10.7 & 1.2 \\
\hline IGR J17597-2201 & $175946-2201.8(30)$ & $175945.7-220139(4)$ & $17594556-2201435$ & 12.9 & 4.9 \\
\hline IGR J18027-2016 & $180239-2017.6(35)$ & $180242.0-201718(4)$ & 18024194-2017172 & 11.4 & 1.1 \\
\hline & & & $180242.0-201720.2$ & & 3.0 \\
\hline \multicolumn{6}{|c|}{ TRANSIENT SOURCES } \\
\hline IGR J16465-4507 & $164630-4507.5(120)$ & $164635.5-450704(4)$ & $16463526-4507045$ & 9.8 & 2.6 \\
\hline IGR J17544-2619 & $175424-2619.0(120)$ & $175425.4-261953(4)^{5}$ & $17542527-2619526$ & 8.0 & 1.7 \\
\hline
\end{tabular}

References: ${ }^{1}$ Schartel et al. (2003); ${ }^{2}$ Rodriguez et al (in prep.); ${ }^{3}$ Bodaghee et al. (2005); ${ }^{4}$ Zurita Heras et al. (2005); ${ }^{5}$ González-Riestra et al. (2004).

IGR J16479-4514 has been detected with a low flux by XMM-Newton (see Sect. 3.3) and is not detected by INTEGRAL in many revolutions indicating that it is highly variable, and usually faint.

The two transient sources were observed by XMM-Newton in TOO mode close to the flaring periods detected by INTEGRAL:

- IGR J16465-4507 was detected during a week of the fourth visibility period. During that time, its average count rate was about 10 times higher than the average upper limit over the first two visibility periods, suggesting that the source is transient.

- IGR J17544-2619 was detected for less than 3 days during each of the first 3 visibility periods. These detections are separated by $56 \pm 1$ and $54 \pm 1$ spacecraft revolutions indicating that it is probably a transient system with a period of $165 \pm 3$ days.

Orbital periods are known in IGR J17252-3616 (9.72 d - Zurita Heras et al. 2005) and in IGR J18027-1455 (4.57 d - Hill et al. 2005). We searched the ISGRI lightcurves for long period signals and found additional signature in a single source: IGR J16320-4751. Figure 3 displays the Lomb-Scargle periodogram of IGR J16320-4751 that features a period of $8.99 \pm$ $0.05 \mathrm{~d}$ and the folded lightcurve that indicates an orbital modulation with a pulse fraction of $70 \pm 4 \%$ without any evidence for an eclipse. Those results are in agreement with the recent SWIFT/BAT results (Corbet et al. 2005).

\subsection{High-energy spectra}

Simultaneous fits were performed with the average XMM-Newton EPIC pn and INTEGRAL IBIS ISGRI spectra extracted as described in Sect. 2. The EPIC spectra were rebinned to oversample the $F W H M$ of the energy resolution and to have a minimum of 20 counts per bin to allow use of the $\chi^{2}$ statistic. The photo-electric absorption cross sections of Morrison \& McCammon (1983) are used throughout. All spectral uncertainties are given at $90 \%$ confidence.

The X-and $\gamma$-ray spectra derived from the XMM-Newton and INTEGRAL observations are very similar among the studied sources (Fig. 4). The continuum emission is steep and
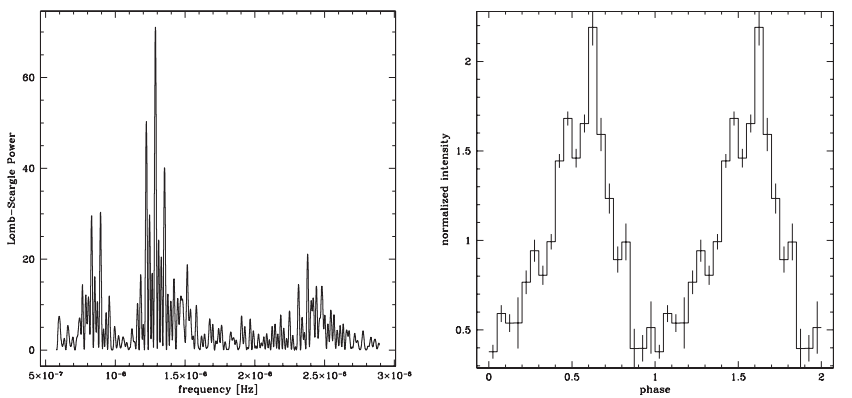

Fig. 3. ISGRI ( $>20 \mathrm{keV}$ ) periodogram of IGR J16320-4751 (left) and folded lightcurve (right) with a period of 8.99 day (phase $0=$ MJD 53512.05).

requires a spectral break above $20 \mathrm{keV}$ when the statistic is good enough. The continuum is absorbed below $10 \mathrm{keV}$ with photoelectric absorbing column densities between $1.8 \times 10^{22}$ and $2 \times 10^{24}$ atom $\mathrm{cm}^{-2}$, much larger than the typical interstellar column density in the plane of the Galaxy excepting for one transient source (listed in Table 6). When the absorbing column density is large enough, (and enough data were collected) strong fluorescence Fe lines are evident in the XMM-Newton EPIC spectra.

\subsubsection{Continuum modeling}

The low energy curvature and the very steep spectra observed at high energy cannot be represented by Compton reflection. The geometry of the sources is therefore characterized by transmission rather than reflection, as already established for IGR J16318-4848 (Matt \& Guainazzi 2003).

The spectral break could be modeled with a cutoff powerlaw or a broken power-law continuum model. However, in those cases very hard power-law slope (even positive photon index) are often obtained that are highly correlated to the absorbing column density. To minimize such effects and allow a comparison of the source parameters that is less model dependent, we decided to use an thermal inverse Comptonization continuum model.

The continuum was modified by an intrinsic photoelectric absorption (with $\mathrm{Fe}$ abundances left free when needed). We also added an $\mathrm{Fe} \mathrm{K} \alpha$ fluorescence line (F $\mathrm{K} \beta$ and $\mathrm{Ni} \mathrm{K} \alpha$ were added 


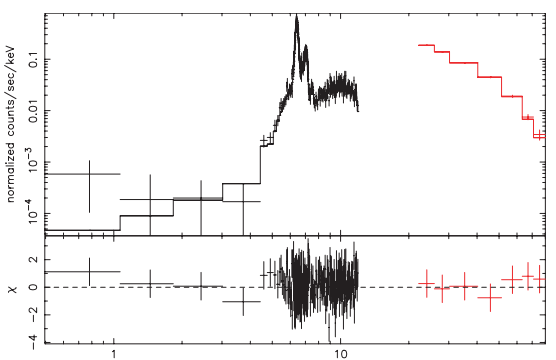

(a) IGR J16318-4848

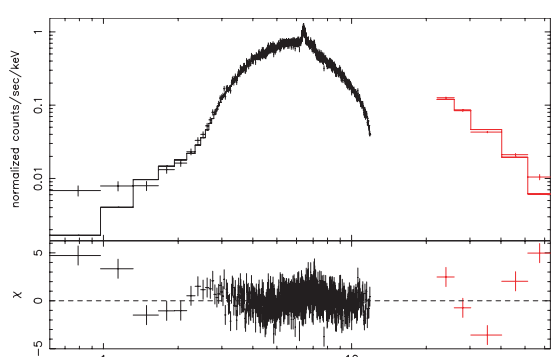

(d) IGR J16320-4751

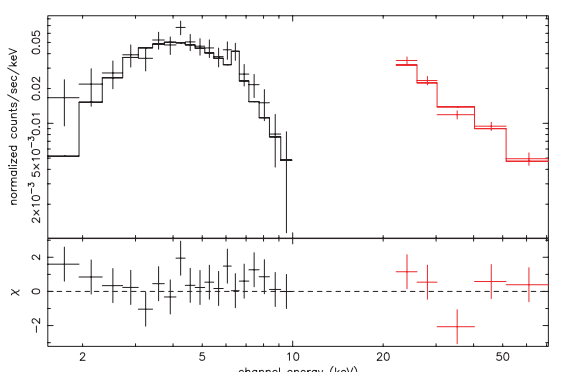

(g) IGR J16479-4514

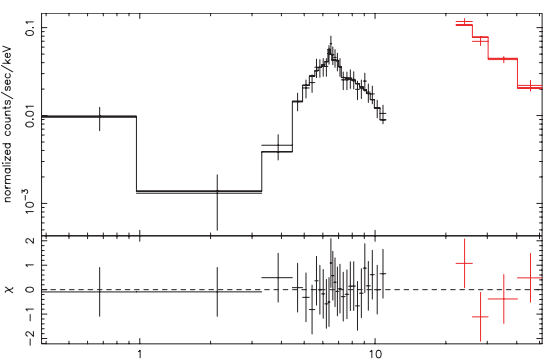

(b) IGR J16465-4507

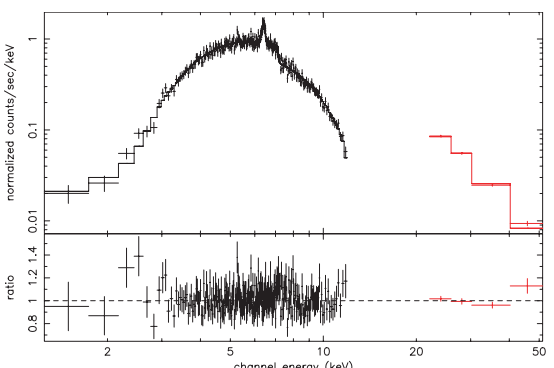

(e) IGR J17252-3616

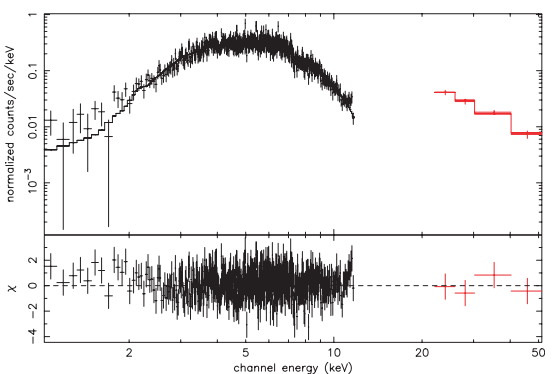

(h) IGR J18027-2016

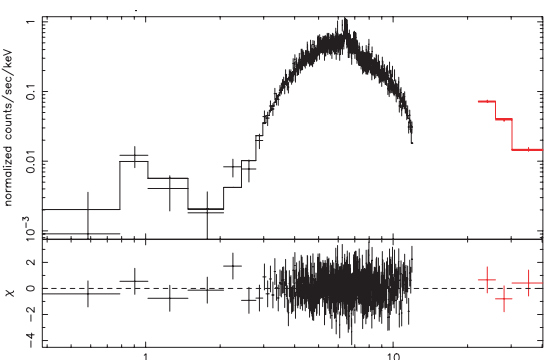

(c) IGR J16393-4641

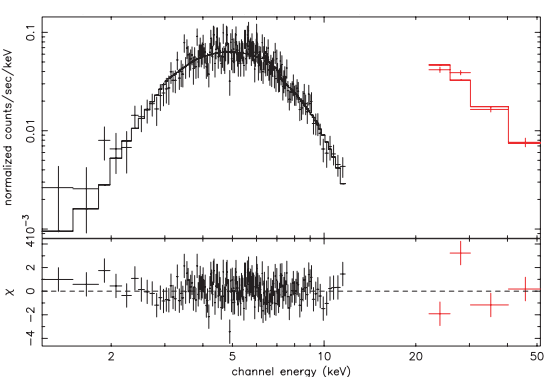

(f) IGR J16418-4532

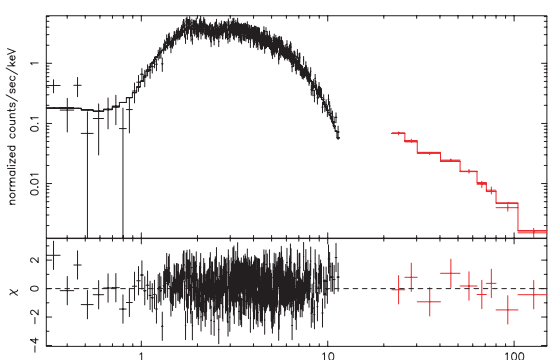

(i) IGR J17597-2201

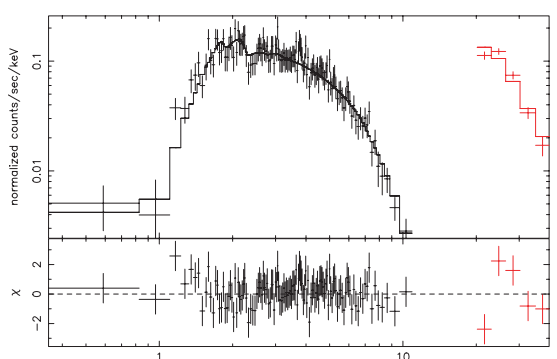

(j) IGR J17544-2619

Fig. 4. ISGRI ( $>20 \mathrm{keV})$ and EPIC $(<15 \mathrm{keV})$ source count spectra with best fit model predictions and residuals. The spectral model, identical in all sources, is described in the text. The sources are ordered from highest to lowest intrinsic absorption.

in a few cases) modeled as a Gaussian, and, when required by the data, a soft X-ray excess absorbed by the galactic column density in the direction of the source derived from the measurements by Dickey \& Lockman (1990). Table 4 lists the main spectral parameters obtained for each source.

The column densities are larger than the galactic absorption by factors of 4 to 100 except for IGR J17544-2619 (GonzálezRiestra et al. 2004). The depth of the Iron edge is fully compatible with the absorbing column density (requiring a slight over or under abundance in four sources).

A normalisation constant was introduced in the model to account for source variability between the XMM-Newton observations and the average source flux measured by INTEGRAL. This constant is compatible with the typical variability observed in those sources. In the case of IGR J16479-4514 and the two transient sources the normalisation constant is large illustrating the strong variability of those sources on short time scales.

The continuum parameters are constrained in a narrow range with typical values found in X-ray pulsars (Nagase 2002).

\subsubsection{Soft excess}

Significant soft X-ray (0.5-2 keV) excesses (listed in Table 4) are found above the low energy extrapolation of the absorbed continuum in five sources. This emission was modeled with a blackbody emission modified by the fixed galactic absorption (the data are not good enough to determine a temperature). Except for IGR J16318-4848, soft X-ray excesses are detected in all sources with absorbing column densities in excess of $10^{23}$ atom $\mathrm{cm}^{-2}$. This is a detection bias as weak soft X-ray 
Table 4. Parameters resulting from the spectral modeling of the EPIC pn and ISGRI spectra of the sources. The xspec spectral model is constant*wabs*(bbody+gaussian+vphabs*compTT). The galactic absorption is fixed. $\chi_{v}^{2}$ is the goodness of fit; $C_{\mathrm{I}}$ is the ISGRI normalization factor; $N_{\mathrm{H}}$ is the intrinsic absorbing column density; $Z_{\mathrm{Fe}}$ is the relative Fe abundance, if needed to fit the Iron edge; $k T_{\mathrm{e}}$ and $\tau$ are the electronic temperature and optical depth of the Comptonizing medium; $E W_{\mathrm{K} \alpha}^{\mathrm{UA}}$ is the equivalent width of the Fe $\mathrm{K} \alpha$ line measured with reference to the unabsorbed intrinsic continuum. Integrated fluxes are listed for the ISGRI spectrum normalisation for both the continuum as observed $\left(F_{2-100}\right)$ and corrected $\left(F_{2-100}^{\mathrm{UA}}\right)$ for the intrinsic photoelectric absorption. The observed flux of the soft excess component $\left(F_{0.5-2}^{\mathrm{SE}}\right)$ is listed separately.

\begin{tabular}{|c|c|c|c|c|c|c|c|c|c|c|}
\hline Source & $\chi_{v}^{2}$ & $\bar{C} C_{\mathrm{I}}$ & $\begin{array}{c}N_{\mathrm{H}} \\
{\left[10^{22} \mathrm{~cm}^{-2}\right]}\end{array}$ & $\begin{array}{l}Z_{\mathrm{Fe}} \\
{\left[Z_{\odot}\right]}\end{array}$ & $\begin{array}{c}k T_{\mathrm{e}} \\
{[\mathrm{keV}]}\end{array}$ & $\tau$ & $\begin{array}{c}E W_{\mathrm{K} \alpha}^{\mathrm{UA}} \\
{[\mathrm{eV}]}\end{array}$ & $\begin{array}{l}F_{2-100} \\
{\left[10^{-10}\right.}\end{array}$ & $\begin{array}{c}F_{2-100}^{\mathrm{UA}} \\
\left.\mathrm{erg} / \mathrm{cm}^{2} \mathrm{~s}\right]\end{array}$ & $\begin{array}{c}F_{0.5-2}^{\mathrm{SE}} \\
{\left[\mathrm{erg} / \mathrm{cm}^{2} \mathrm{~s}\right]}\end{array}$ \\
\hline \multicolumn{11}{|c|}{ PERSISTENT SOURCES } \\
\hline IGR J16318-4848 & 0.94 & 1.2 & $193 \pm 11$ & 0.88 & $8.5 \pm 0.4$ & $3.8_{-0.2}^{+0.7}$ & $82 \pm 3$ & 3.5 & 8.0 & $<4 \times 10^{-16}$ \\
\hline IGR J16320-4751 & 1.35 & 0.55 & $18.3 \pm 0.7$ & 0.74 & $5.6 \pm 0.2$ & $7.9 \pm 0.5$ & $85 \pm 5$ & 2.5 & 2.8 & $85 \times 10^{-15}$ \\
\hline IGR J16393-4641 & 0.92 & 0.76 & $23.7 \pm 0.7$ & & $4.4 \pm 0.4$ & $10.1 \pm 0.4$ & $60 \pm 10$ & 1.7 & 2.1 & $8 \times 10^{-15}$ \\
\hline IGR J16418-4532 & 0.98 & 4.7 & $10.0 \pm 1.2$ & & $6.0 \pm 0.5$ & $5.5 \pm 0.7$ & $<34$ & 1.1 & 1.3 & $<7 \times 10^{-15}$ \\
\hline IGR J16479-4514 & 1.22 & 19.3 & $7.7 \pm 1.7$ & & $>13$ & $<1.8$ & $<280$ & 1.4 & 1.8 & $<35 \times 10^{-15}$ \\
\hline IGR J17252-3616 & 1.04 & 0.44 & $14.9 \pm 1.2$ & 1.5 & $5.6 \pm 0.2$ & $7.9 \pm 0.5$ & $84 \pm 11$ & 1.8 & 2.2 & $26 \times 10^{-15}$ \\
\hline IGR J17597-2201 & 1.00 & 0.49 & $4.5 \pm 0.7$ & & $>20$ & $<2.0$ & $<10$ & 3.0 & 3.4 & $6 \times 10^{-12}$ \\
\hline IGR J18027-2016 & 0.96 & 0.44 & $9.1 \pm 0.5$ & & $7.2 \pm 0.7$ & $6.6 \pm 0.5$ & $33 \pm 18$ & 1.0 & 1.1 & $<39 \times 10^{-15}$ \\
\hline \multicolumn{11}{|c|}{ TRANSIENT SOURCES } \\
\hline IGR J16465-4507 & 0.39 & 4.3 & $60 \pm 10$ & 0.46 & $7.0 \pm 0.5$ & $6.5 \pm 2.0$ & $<60$ & 2.1 & 2.8 & $18 \times 10^{-15}$ \\
\hline IGR J17544-2619 & 0.91 & 61 & $1.8 \pm 0.3$ & & $5.9 \pm 0.6$ & $3.8 \pm 0.5$ & $<35$ & 5.5 & 6.1 & $<17 \times 10^{-14}$ \\
\hline
\end{tabular}

excesses cannot be detected if the primary continuum is not absorbed.

Soft X-ray excesses have been resolved in high resolution spectra of supergiant HMXB, especially during eclipse, and have been interpreted as fluorescent and recombination lines and/or scattering in the outer parts of the systems (van der Meer et al. 2004; Sako et al. 2002).

The soft excesses observed in our sources are probably of similar nature, however excesses in low resolution spectra could also be represented by partial covering or hot absorber (e.g. Kuulkers 2005) models. In all sources featuring a soft X-ray excess, we have tried an alternate model based on a partial covering absorber.

In three sources, the soft X-ray excess could be mostly explained by partial covering of the intrinsic absorption: (1) in IGR J17252-3616, a covering factor of $0.995 \pm 0.002$ (Zurita Heras et al. 2005) could explain satisfactorily the soft X-ray excesses; (2) in IGR J16320-4751, a covering factor of $0.998 \pm$ 0.002 could explain most of the excess. In that case, a small excess $\left(F_{0.5-2 \mathrm{keV}}=5 \times 10^{-15} \mathrm{erg} \mathrm{cm}^{-2} \mathrm{~s}^{-1}\right)$ and galactic absorption $\left(N_{\mathrm{H}}=1.1 \times 10^{22}\right.$ atom $\left.\mathrm{cm}^{-2}\right)$ lower than the value derived from (Dickey \& Lockman 1990) are however required to obtain a good fit of the data; (3) in IGR J17597-2201, a covering factor of $0.90 \pm 0.02$ could represent the X-ray spectrum without any soft excess component $\left(\chi_{v}^{2}=1.0\right)$. In this case, the resulting intrinsic absorbing column density $N_{\mathrm{H}}=$ $(2.70 \pm 0.15) \times 10^{22}$ atom $\mathrm{cm}^{-2}$ differs significantly from the value listed in Table 4.

Partial covering opens the possibility to observe a Compton reflection continuum at high energy in the form of a hard energy tail or of an additional flat low energy continuum. There is, however, no evidence for a high energy tail and the spectral shape of the soft excesses do not correspond to what would be expected from Compton reflection.

In two sources, the soft X-ray excess cannot be accounted for with a simple partial covering absorber model: (1) in IGR J16465-4507, the soft X-ray excess is very large and a partial covering model increases the $\chi^{2}$ by 13 for 29 d.o.f.; (2) in IGR J16393-4641 partial covering cannot explain the observed spectrum, a soft excess remains needed (Bodaghee et al. 2005).
The observed soft X-ray excesses could therefore be explained by various spectral models. Better spectral resolution and statistics would be needed to disentangle them. It however seems likely that the excesses observed in HMXB during eclipse could also explain the excesses detected when most of the continuum emission is absorbed. This interpretation also suggests that the absorbing matter is located close to the compact object.

\subsubsection{Fe $\mathrm{K} \alpha$ line}

The centroid of the $\mathrm{Fe} \mathrm{K} \alpha$ line, compatible with $6.4 \mathrm{keV}$, and the width of the lines, compatible with zero, in all cases indicate that the fluorescence lines are emitted in gas that is not strongly ionized.

The apparent $\mathrm{Fe} \mathrm{K} \alpha$ line equivalent width is strongly variable from source to source (between less than $100 \mathrm{eV}$ to more than $2 \mathrm{keV}$ ). However, when compared to the continuum corrected for the effect of the intrinsic photoionisation absorption the equivalent width reduces to 30 to $90 \mathrm{eV}$ in all intrinsically absorbed sources. This indicates that fluorescence could originate from the same gas configuration among the studied sources with the gas surrounding the X-ray source.

\subsection{Pulsations}

Hard X-ray pulsation as a general characteristic of new persistent INTEGRAL sources has been reported by Walter (2004). Pulsation has been reported in detailed studies of several of our sample sources (Bodaghee et al. 2005; Zurita Heras et al. 2005; Lutovinov et al. 2005b; Hill et al. 2005). No pulsation has been found in IGR J16318-4848.

Here, we report on the remaining individual sources of our sample. A summary of the pulsation periods is given in Table 6 . Pulsations were also searched for below $1.5 \mathrm{keV}$ in sources featuring a soft X-ray excesses to disentangle independent soft $\mathrm{X}$-ray excess from partial covering absorber models. The data are unfortunately not good enough to reach a definite conclusion. 

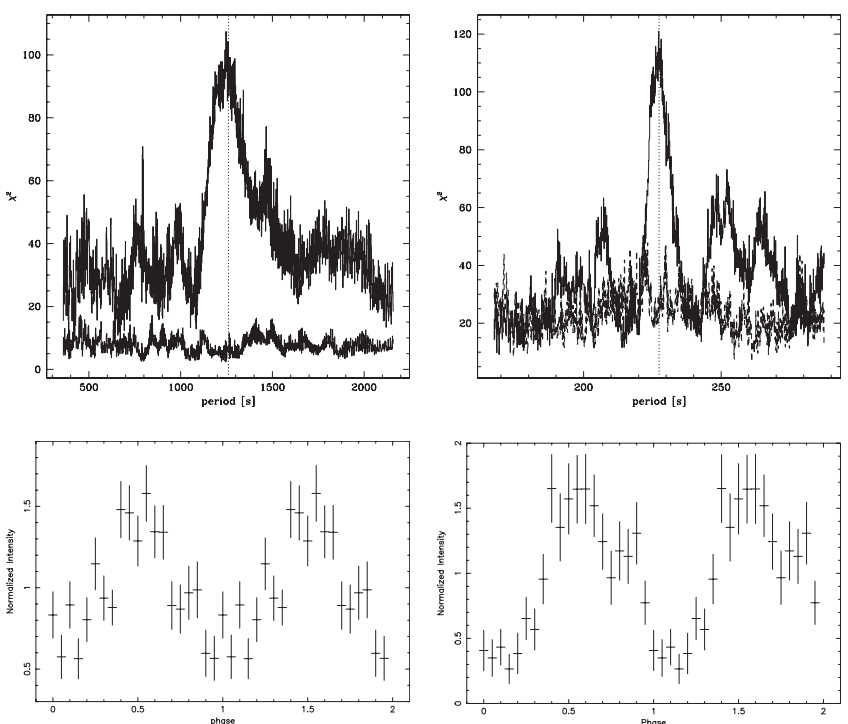

Fig. 5. XMM EPIC pn $\chi^{2}$ periodogram (lower curve is derived from the background) and folded lightcurve for IGR J16418-4532 (left) and IGR J16465-4507 (right).

\subsubsection{IGR J16418-4532}

Removing the periods with strong background flares resulted in $10.5 \mathrm{ks}$ of good EPIC data. A $30 \mathrm{~s}$ resolution lightcurve was extracted. During the first half of the observation the source featured 3 short and strong flares reaching about 2 count $\mathrm{s}^{-1}$ while the averaged count rate is about 0.2 count s ${ }^{-1}$ for most of the observation. To search for a period in the quiescent state, intervals of time affected by flares were removed from the dataset which resulted in a continuous $9 \mathrm{ks}$ lightcurve. We searched for a period by folding the data through ranges of periods and compared the results with the ones obtained from the background lightcurve (Fig. 5).

A period of $1246 \pm 100 \mathrm{~s}$ is present in the data. The probability of chance detection can be estimated as below $10^{-3}$. The pulse fraction (Fig. 5) is $64 \pm 10 \%(2-10 \mathrm{keV})$.

The source is too faint to search for a period with ISGRI and folding the ISGRI 15-30 keV lightcurve of the source obtained on revolution 37 (MJD 52671.08-52674.07) using a period of $1246 \mathrm{~s}$ did not provide any significant result.

\subsubsection{IGR J16479-4514}

The XMM-Newton observation of IGR J16479-4514 was affected by a very strong and variable background. The low source count rate when compared to the strong background is such that the lightcurve is completely dominated by the background variations. No period could be searched for.

We also searched a $100 \mathrm{~s}$ resolution ISGRI lightcurve of revolution 100 (MJD 52 859.48-52862.47), when the source was bright, but no pulsation was found.

\subsubsection{IGR J16465-4507}

A $10 \mathrm{~s}$ resolution EPIC (2-10 keV) lightcurve was extracted.

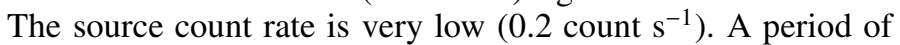
$227 \pm 5 \mathrm{~s}$ is found in the lightcurve and absent from the background (Fig. 5). The probability of chance detection can be estimated as below $10^{-3}$. The pulse fraction (Fig. 5) is $80 \pm 16 \%$. A lightcurve folded with the same period was extracted at low

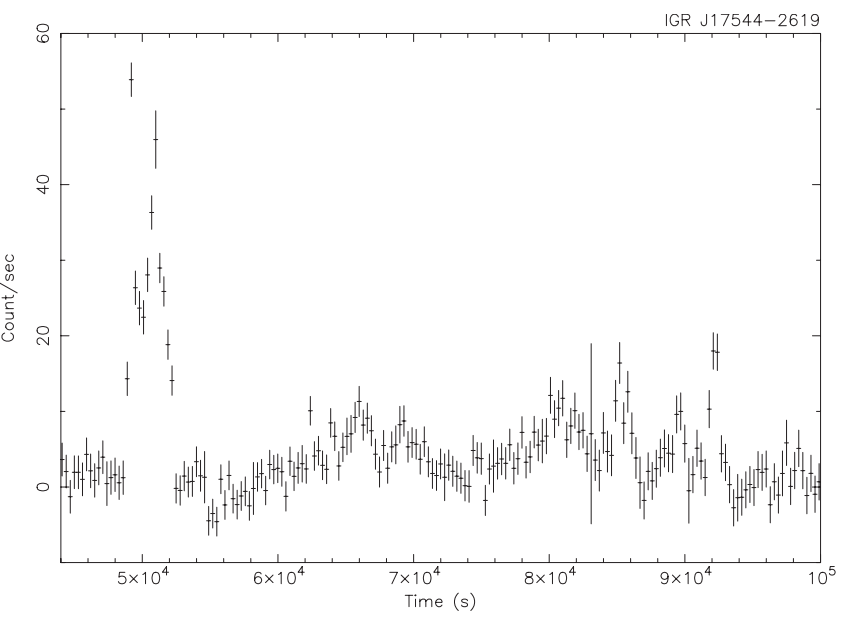

Fig. 6. INTEGRAL/ISGRI 15-30 keV lightcurve of IGR J17544-2619.

energy $(0.4-1.5 \mathrm{keV})$ to study the strong soft X-ray excess found in this source but the number of counts was too low to reach any conclusion.

We also searched a $10 \mathrm{~s}$ time bin ISGRI lightcurve of revolution 232, when the source was bright, but no pulsation was found.

EPIC pulse phase spectra have been built for the pulse $(0.38-$ $0.92)$ and the interpulse $(0.92-0.38)$ phase regions. The spectra do not show significant spectral shape variations and could be fitted with the parameters listed in Table 4 besides the change in continuum normalisation $\left(\chi_{v}^{2}=1.05\right)$.

\subsubsection{IGR J17544-2619}

The XMM-Newton observation was presented by GonzálezRiestra et al. (2004). No pulsation was detected but the source featured a number of short flares. During the 3 INTEGRAL revolutions when the source was bright, the ISGRI lightcurve shows intense variability with count rate increasing from $0 \pm 15$ to $80 \pm 15$ count $\mathrm{s}^{-1}(\approx 1 \mathrm{Crab})$ in $300 \mathrm{~s}(15-30 \mathrm{keV})$. Figure 6 shows the strong source activity that occured during revolution 113 (MJD 52 898.36-52901.35) just before the XMM-Newton observation. Study of those flares did not reveal any periodicity.

\subsubsection{IGR J17597-2201}

The XMM-Newton observation of IGR J17597-2201 was affected by on-board buffer overflow due to high background and to the strength of the source, generating an instrumental periodicity of $192 \mathrm{~s}$. The EPIC pn lightcurve suggests a period of $1800 \mathrm{~s}$ that is however not significant. Nothing could be found in the ISGRI data.

\subsection{Infrared photometry}

We have collected (see Table 5) $J H K$ magnitudes available from the 2MASS catalogue (Cutri et al. 2003) for the candidate stellar counterparts (see Sect. 3.1), and in some cases additional BRI magnitudes from the USNO and GSC catalogues (these data were collected through the VizieR service (Ochsenbein et al. 2000).

In order to derive an upper limit on the reddening, we fitted these data with a reddened blackbody model following a procedure detailed in Filliatre \& Chaty (2004). The temperature of 
Table 5. Counterpart photometry. $J H K$ magnitudes are from the 2MASS catalogue. BRI magnitudes are from the USNO and GSC catalogue (obtained through Vizier) and from Filliatre \& Chaty (2004) for IGR J16318-4848.

\begin{tabular}{lcccrrr}
\hline \hline Counterpart & $B$ & $R$ & $I$ & $J$ & \multicolumn{1}{c}{$H$} & \multicolumn{1}{c}{$K$} \\
\hline $16314831-4849005$ & $25.4 \pm 1.0$ & $17.7 \pm 0.1$ & $16.1 \pm 0.5$ & $10.16 \pm 0.02$ & $8.33 \pm 0.03$ & $7.19 \pm 0.02$ \\
$16320175-4752289$ & & & & $14.08 \pm 0.10$ & $13.03 \pm 0.05$ & $10.99 \pm 0.03$ \\
$16390535-4642137$ & & & & $14.63 \pm 0.06$ & $13.32 \pm 0.04$ & $12.78 \pm 0.04$ \\
$16415078-4532253$ & & & & $13.87 \pm 0.05$ & $12.31 \pm 0.04$ & $11.48 \pm 0.03$ \\
$16480656-4512068$ & & 18.4 & 16.7 & $12.95 \pm 0.03$ & $10.83 \pm 0.02$ & $9.80 \pm 0.02$ \\
$17251139-3616575$ & & & & $14.23 \pm 0.03$ & $11.81 \pm 0.03$ & $10.67 \pm 0.02$ \\
$17594556-2201435$ & & & & $14.87 \pm 0.07$ & $13.44 \pm 0.07$ & $12.95 \pm 0.07$ \\
$18024194-2017172$ & & & & $12.72 \pm 0.04$ & $11.91 \pm 0.04$ & $11.47 \pm 0.03$ \\
$180242.0-201720.2$ & 17.5 & 15.5 & 14.2 & $12.87 \pm 0.04$ & $13.30 \pm 0.04$ & $11.75 \pm 0.03$ \\
$16463526-4507045$ & $15.2 \pm 0.3$ & $13.2 \pm 0.3$ & $11.7 \pm 0.3$ & $10.54 \pm 0.03$ & $10.08 \pm 0.03$ & $9.84 \pm 0.02$ \\
$17542527-2619526$ & 14.2 & $11.5 \pm 0.4$ & 10.4 & $8.79 \pm 0.02$ & $8.31 \pm 0.03$ & $8.02 \pm 0.03$ \\
\hline
\end{tabular}

Table 6. Properties of the newly discovered INTEGRAL sources that have been observed with sensitive X-ray instruments. $N_{\mathrm{H}}^{\text {gal }}$ is the galactic column in the source direction (Dickey \& Lockman 1990). $N_{\mathrm{H}}^{E_{B-V}}$ is the maximum absorption derived from the infrared photometry. $N_{\mathrm{H}}$ is the measured intrinsic X-ray absorbing column density. $P_{\text {spin }}$ is the spin period in sec and $P_{\text {orb }}$ the orbital period in days.

\begin{tabular}{|c|c|c|c|c|c|c|c|}
\hline Source & Region & $\begin{array}{c}P_{\text {spin }} \\
{[\mathrm{s}]}\end{array}$ & $\begin{array}{l}N_{\mathrm{H}} \\
{\left[10^{22}\right.}\end{array}$ & $\begin{array}{l}N_{\mathrm{H}}^{\mathrm{gal}} \\
\text { atom }\end{array}$ & $\begin{array}{c}N_{\mathrm{H}}^{E_{B-V}} \\
\left.\mathrm{~cm}^{-2}\right]\end{array}$ & SP. T. & $\begin{array}{c}P_{\text {orb }} \\
{[d]}\end{array}$ \\
\hline \multicolumn{8}{|c|}{ PERSISTENT SOURCES } \\
\hline IGR J16318-4848 & Nor & & 193 & 2.1 & $<4.2$ & $\operatorname{sgB}[e]^{1}$ & \\
\hline IGR J16320-4751 & Nor & $1300^{2}$ & 18 & 2.1 & & Early type ${ }^{8}$ & 8.99 \\
\hline IGR J16393-4641 & Nor & $912^{3}$ & 24 & 2.2 & $<2.7$ & & \\
\hline IGR J16418-4532 & Nor & 1246 & 10 & 2.0 & $<3.6$ & & \\
\hline IGR J16479-4514 & Nor & & 8 & 2.1 & $<4.8$ & Early type $^{8}$ & \\
\hline IGR J17252-3616 & GC & $414^{4}$ & 15 & 1.5 & $<5.3$ & Early type $^{8}$ & $9.72^{4,6}$ \\
\hline IGR J17597-2201 & GC & & 5 & 1.2 & $<2.9$ & Late type $^{8}$ & \\
\hline IGR J18027-2016 & $\mathrm{GC}$ & $139^{5}$ & 9 & 1.1 & $<2.1$ & $\operatorname{sgOB}^{5}$ & $4.57^{5}$ \\
\hline \multicolumn{8}{|c|}{ TRANSIENT SOURCES } \\
\hline IGR J16465-4507 & Nor & 228 & 60 & 1.6 & $<1.0$ & $\operatorname{sgB}^{7}$ & \\
\hline IGR J17544-2619 & GC & & 2 & 1.4 & $<2.5$ & $\operatorname{sgB}^{8}$ & \\
\hline
\end{tabular}

References: ${ }^{1}$ Filliatre \& Chaty (2004); ${ }^{2}$ Lutovinov et al. (2005b) $;{ }^{3}$ Bodaghee et al. (2005); ${ }^{4}$ Zurita Heras et al. (2005); ${ }^{5}$ Hill et al. (2005); ${ }^{6}$ Markwardt \& Swank (2003); ${ }^{7}$ Smith (2004); ${ }^{8}$ Chaty et al (in prep.).

the black-body was fixed to $20000 \mathrm{~K}$ to insure that only the Rayleigh-Jeans part of the spectrum contributes in the available spectral band. If the star is intrinsically cooler or if it harbors any additional infrared contribution (from dust/disk), the absorption must be lower.

We checked that the normalizations resulting from the spectral fits are consistent with the luminosity of a bright blue star given a distance range of $1-10 \mathrm{kpc}$. In the case of IGR J16320-4751, we were unable to derive reasonable fits. Possible problems may include strong departures from the blackbody model and photometric errors. The upper limit on the reddening obtained for IGR J16318-4848 is fully in agreement with the value derived by Filliatre \& Chaty (2004).

The reddening upper limits were converted to $N_{\mathrm{H}}$ according to Predehl \& Schmitt (1995) and listed in Table 6. These upper limits are consistent with the galactic interstellar absorption in the direction of the sources and significantly smaller than the amount of absorption derived from the X-ray data excepting for IGR J17597-2201 and IGR J17544-2619. Such conclusions, already derived for IGR J16318-4848 (Walter et al. 2003b; Filliatre \& Chaty 2004) and IGR J16320-4751 (Rodriguez et al. 2003), indicate that, excepting for IGR J17597-2201 and
IGR J17544-2619, the absorbing matter is not interstellar but concentrated close to the X-ray sources.

\section{Discussion}

Among the unidentified sources detected by INTEGRAL in the galactic plane, XMM-Newton observations could be obtained for 10 objects. Eight sources appear to be persistent, two are transient.

Using the precise positions provided by XMM-Newton, we found bright infrared candidate counterparts in the 2MASS survey images for all sources. The upper limits on the absorption derived from the available photometry are consistent with the galactic interstellar absorption and smaller than the absorption derived from the X-ray data for eight sources. In those sources the additional absorption of the X-ray sources is local to the compact source and not of interstellar origin. More precisely the absorbing matter is located close enough to the source so that dust could not form.

In our sample, seven sources are persistent and feature absorbing column densities much larger than the galactic value. Those sources are discussed in Sects. 4.2 to 4.4. The three 
sources that do not share those properties are discussed in Sect. 4.1.

\subsection{Transient or unabsorbed sources}

IGR J17544-2619 could be a long period (165 d) system flaring at periastron passage. The system did not show any enhanced absorption and shares the properties of typical Be systems. During flares, the source flux became 100 times brighter than the typical flux observed in the persistent sources. This suggests accretion events reaching the Eddington limit typically observed in Rochelobe overflow systems when the compact object reaches the periastron of an eccentric orbit. The early spectral type (Chaty et al., in prep.) of the infrared counterpart we suggest confirms the HMXB nature of the source.

IGR J16465-4507 is a transient X-ray pulsar featuring huge absorbing column density (when active). The companion of IGR J16465-4507 is a supergiant massive star (Smith 2004). This object could be a long orbital period system similar to IGR J17544-2619.

IGR J17597-2201 is a persistent source that is not strongly absorbed (the additional column density is compatible with the upper limit on the infrared reddening). This system has been proposed to be a LMXB (Lutovinov et al. 2005a) which is in agreement with the late spectral type of the infrared counterpart we have proposed (Chaty et al, in prep.).

\subsection{Persistent intrinsically absorbed sources}

The seven persistent absorbed sources in our sample are bright $\left(>10^{-10} \mathrm{erg} \mathrm{s}^{-1} \mathrm{~cm}^{-2}\right.$ ) above $10 \mathrm{keV}$ but weak enough in the $\mathrm{keV}$ range to have remained largely unnoticed in previous X-ray galactic plane surveys. In some cases, they could have been missed also because of their variability on time scales of hours to days.

A major step forward in understanding these systems has come from the detection of X-ray pulsations in the source light curves. Pulse periods ranging from 139 to $1300 \mathrm{~s}$ have been determined for five of the seven sources. No pulsation was found from IGR J16479-4514 and in IGR J16318-4848. In the former object, the presence of a pulsar is not excluded as the XMM-Newton and ISGRI data, that were of too low quality to allow any period detection, are compatible with a pulsed fraction of $100 \%$ for a period of $1 \mathrm{ks}$. In the latter source, either the pulse fraction is small $(<10 \%)$, or the spin period is very long $\left(P_{\mathrm{s}}>5 \mathrm{ks}\right)$.

Known HMXBs accreting pulsars with pulse periods exceeding $100 \mathrm{~s}$ fall into two distinct groups according to the nature of their companion stars. The Be binary systems with orbital periods above 100 days and the supergiant systems with shorter orbital periods. In supergiant systems, Waters \& van Kerkwijk (1989) have suggested that the long spin periods resulting from the propeller effect (Illarionov \& Sunyaev 1975) during the post SN evolution could remain during the high-mass X-ray binary phase of the system almost independently of the orbital period.

Among the persistent INTEGRAL sources, estimates of the orbital period are only available for four systems: $4.57 \mathrm{~d}$ for IGR J18027-1455 (Hill et al. 2005), $8.99 \mathrm{~d}$ for IGR J16320-4751, 9.7 d for IGR J17252-3616 (Markwardt \& Swank 2003) and $13 \mathrm{~d}$ for IGR J19140+0951 (Corbet et al. 2004) that are therefore very probably supergiant systems. Two additional persistent sources of our sample have spin periods

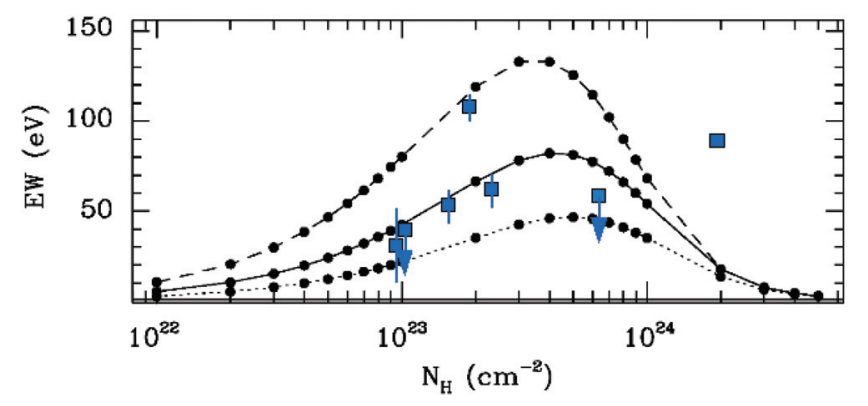

Fig. 7. The Fe K $\alpha$ equivalent width calculated with reference to the unabsorbed continuum (and corrected for the peculiar Iron abundances when needed) plotted against the absorbing column density. The curves show the prediction of a spherical distribution of matter around the X-ray source for different Fe abundances (from Matt 2002).

consistent with such systems. Those sources being persistent they are unlikely to be Be systems and likely supergiant as well.

The observed X-ray spectral shape clearly point to a transmission geometry where the compact source is embedded in dense material (a reflection geometry cannot explain the spectra). When compared to the continuum flux corrected for the photoelectric absorption, the strength of the Fe fluorescence line varies in a narrow range $(30-90 \mathrm{eV})$ indicating that a very significant fraction of the solid angle around the X-ray sources is filled with cold matter. The relation between the $\mathrm{Fe} \mathrm{K} \alpha$ equivalent width and the absorbing column density could help understanding the geometry of the cold dense matter surrounding the X-ray sources. Our data, corrected for the peculiar Fe abundances when needed, are displayed in Fig. 7 together with the results of simulations obtained by Matt (2002) for a spherical shell geometry. Except for IGR J16 318-4848, which displays an excess of absorption on the line of sight, all sources are compatible with a spherical distribution of dense gas around the X-ray sources.

The excess of absorption on the line of sight in IGR J16318-4848 could be related to the absence of soft X-ray excess in that source (Sect. 3.3.2) and to the B[e] type companion. In this source most of the absorption could be related to matter located far away from the neutron star, e.g. the cold circumstellar matter responsible for the $\mathrm{B}[\mathrm{e}]$ low ionization spectral line, that contributes less to the fluorescence.

The picture emerging of these observations is that of a neutron star (or a black hole in some cases), orbiting constantly in dense material expelled from a supergiant stellar companion. Spectral identification of the infrared counterparts is ongoing. 5 out the 7 counterparts show early spectral type or evidence for a high-mass, confirming their HMXB nature (Table 6). Recent observations of the highly-absorbed persistent source IGR J19140+0951 (Rodriguez et al. 2005) are compatible with the above picture.

\subsection{Galactic distribution}

When compared to known HMXBs detected by INTEGRAL, the density of new bright INTEGRAL sources from the catalogue of Bird et al. (2004) peaks in the galactic bulge and in the Norma arm (Walter et al. 2004) regions (Fig. 8). The number of new bright sources detected in the Norma region is significantly higher than the number of new bright sources found in the Scutum arm with a chance probability to be identical lower than $0.1 \%$ (the two regions have similar exposure in the images used by Bird et al. 2004). Interestingly, Bronfman et al. (2000) 


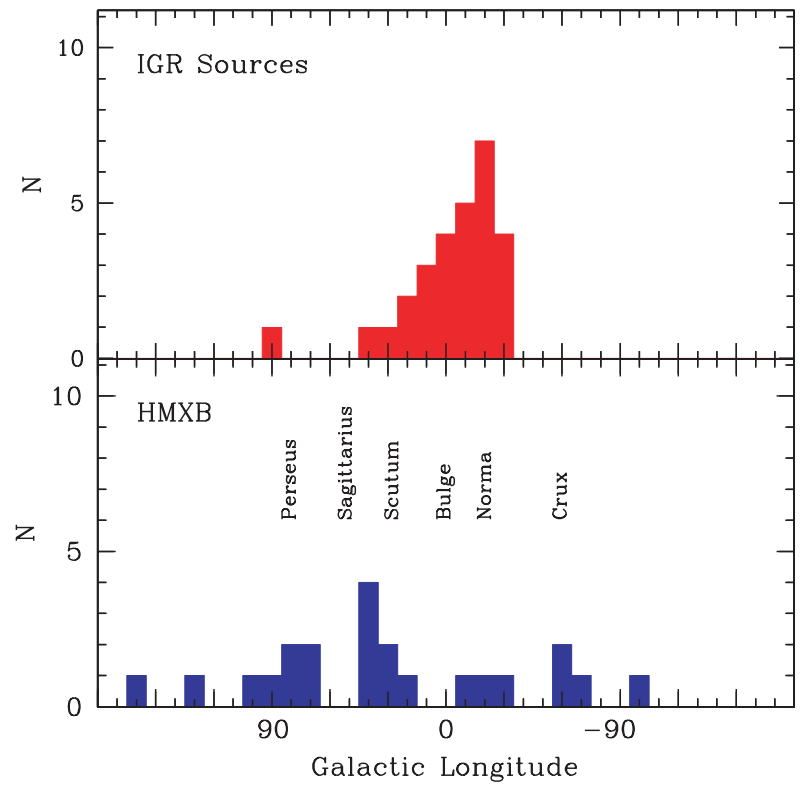

Fig. 8. Galactic longitude distribution of new bright INTEGRAL sources and of known HMXBs detected by INTEGRAL (according to Bird et al. 2004).

reported that the Norma arm is currently the region of the galaxy with the highest OB star formation rate.

Hipparcos observations of supergiant OB systems suggest that those systems have peculiar tangent velocities (corrected for the differential galactic rotation) above $60 \mathrm{~km} \mathrm{~s}^{-1}$ (Chevalier \& Ilovaisky 1998). With an age since SN explosion of few $10^{6}$ years, supergiant HMXB systems could have reached locations as distant as $0.1 \mathrm{kpc}$ from their birth location. The new sources in the Norma region are distributed within 2 degrees of the galactic plane. Such narrow distribution could be kept only if the source's distance is higher than $2 \mathrm{kpc}$. The spread of the Norma source distribution with galactic longitude (about 10 degrees) indicates that these sources most likely did not originate from a single location in the Norma arm.

If the distance to the sources is between 5 and $10 \mathrm{kpc}$, the luminosities that could be inferred from the infrared photometry, $0.3-10 \times 10^{5} L_{\odot}$, is in good agreement with the expectations for 15 to $30 M_{\odot}$ companions. IGR J16318-4848 is significantly brighter in the infrared, however, sgB[e] stars are known to have infrared excesses of several magnitudes (Zickgraf et al. 1992).

\subsection{Geometry}

In HMXB, the absorbing column density expected from a spherically symetric stellar wind (terminal velocity $V_{\mathrm{W}} \approx$ $1000 \mathrm{~km} \mathrm{~s}^{-1}$ ) of the companion (of radius $R_{\mathrm{C}} \approx 10^{12} \mathrm{~cm}$ ) can be evaluated as

$N_{\mathrm{H}} \approx \frac{\dot{M}}{4 \pi R_{\mathrm{C}} V_{\mathrm{W}} m_{\mathrm{H}}} \approx 2 \times 10^{22}$ atom $\mathrm{cm}^{-2} \frac{\dot{M}}{10^{-6} M_{\odot} \mathrm{yr}^{-1}}$.

This is too small to account for the observation unless the strongly absorbed systems feature extreme mass loss rates, small wind velocities or tidal accretion streams (Blondin et al. 1991).

For small orbital radii, the Stomgren sphere around the X-ray source $\left(R_{\mathrm{S}} \approx R_{\mathrm{C}}\right)$ will efficiently photoionize the stellar wind such that the acceleration of the wind will be very much reduced. Reduction of the wind velocity by a factor of 5 could increase the absorbing column density to more than
$10^{23}$ atom $\mathrm{cm}^{-2}$ (Blondin 1994) forming an accretion wake/torus of dense material surrounding the compact object. In the absence of eclipse, for a face-on orbit and a relatively low X-ray luminosity $\left(\approx 10^{36} \mathrm{erg} \mathrm{s}^{-1}\right)$, the compact source could remain occulted by this shadow wind for a large fraction of the orbit. In eclipsing systems like IGR J17252-3616 or IGR J18027-2016 larger variations of the absorbing column density are expected.

The compact object should have an important impact on the wind flow creating inhomogeneities that could be responsible for the strong variability observed on time scales of the order of a fraction of the orbital period. Negueruela (1998) have shown that systems with small orbits, accreting from dense regions of a perturbed disk, will feature X-ray outbursts. Negueruela (2004) suggested a relation between fast transient HMXB systems and high absorption which could indicate a different dynamic of the stellar wind than in the persistent systems.

It is interesting to note that within the proposed geometry, soft X-ray excesses emitted close to the neutron star (surface, accretion flow) would be completely absorbed and therefore not detected. The soft X-ray excesses observed in some of our sources are very weak with $F^{\mathrm{SE}} \approx 10^{-4} F_{2-100}$ and compatible with the expectation from $X$-ray reprocessing in larger photoionised shells (Hickox et al. 2004; Schulz et al. 2002).

In sgB[e] system, like IGR J16318-4848 (Filliatre \& Chaty 2004) or CI Cam (Hynes et al. 2002) dense equatorial wind components have been observed. Models of $\mathrm{B}[\mathrm{e}]$ systems predict the possible co-existence of a rapid highly ionised polar wind with a slow dense, low ionized, equatorial component with an extension of 10-20 stellar radii and a thickness of up to 1 stellar radius $/ 10^{24} \mathrm{~cm}^{-2}$ (Stee 1998). In IGR J16318-4848, the low ionisation of Iron is compatible with a size of the fluorescence region of the order of $10^{8} \mathrm{~km}$ which was also found to be compatible with the absence of delay between $\mathrm{Fe}$ line and continuum flux variations observed at one occasion (Walter et al. 2003b). We therefore suggest that the compact object orbits within the dense wind component of that system explaining the excess of absorbing matter on the line of sight and the absence of a soft $\mathrm{X}$-ray excess.

\section{Conclusions}

Among the 10 unidentified INTEGRAL sources with XMM observations we have found that:

- All sources are detected below $10 \mathrm{keV}$.

- Candidate infrared counterparts have been found for all sources. Their reddening is compatible with the expectation from interstellar absorption.

- IGR J17597-2201 is probably a LMXB with little or no intrinsic absorption. IGR J17544-2619 is a long period (165 d) transient (probably a Be system) without strong intrinsic absorption. IGR J16465-4507 is a highly absorbed supergiant transient system.

- The other 7 sources are persistent, intrinsically absorbed $\left(0.8-19.3 \times 10^{23} \mathrm{~cm}^{-2}\right)$ and very likely supergiant systems. Long pulse periods (139-1300 s) have been found in 5 of these sources, with large pulse fractions. These are X-ray pulsars. One source (IGR J16318-4848) does not feature a strong pulsation or has a period longer than 2-5 ks. In these sources, a transmission geometry is likely with the region emitting fluorescence lines related to the absorbing material.

- Dense shadow wind flows could explain the observed X-ray absorption. Such absorption could be persistent for face-on orbits. The very strong absorption observed in 
IGR J16318-4848 is probably related to a more extended dense stellar wind.

- Previously, only 10 supergiant HMXB were known in the Galaxy (Liu et al. 2000). If a significant fraction of the new sources detected by INTEGRAL are absorbed supergiant wind accretors, as suggested here, then the number of supergiant HMXB could be $2-3$ times higher.

- The density of new bright INTEGRAL sources is significantly enhanced in the direction of the inner Norma arm of the galaxy. As many systems are HMXB, a burst of stellar formation may have occurred recently in that region.

With the accumulation of exposure time and the increased sensitivity that will result from an extended mission lifetime, INTEGRAL is very likely to further increase the number of known persistent (i.e. supergiant) HMXB systems. Extrapolating the $\log N-\log S$ of the unidentified sources predicts the possible detection of up to a hundred supergiant HMXB systems in the galaxy during the next years by INTEGRAL. Identification of these sources will require an intense follow-up programme with XMM-Newton and other missions.

Acknowledgements. Based on observations obtained with the ESA science missions INTEGRAL and XMM-Newton. The INTEGRAL and XMM-Newton instruments and data centers were directly funded by ESA member states and the USA (NASA).

\section{References}

Apparao, K. M. V. 1994, Space Sci. Rev., 69, 255

Augello, G., Iaria, R., Robba, N. R., et al. 2003, ApJ, 596, L63

Bird, A. J., Barlow, E. J., Bassani, L., et al. 2004, ApJ, 607, L33

Blondin, J. M. 1994, ApJ, 435, 756

Blondin, J. M., Stevens, I. R., \& Kallman, T. R. 1991, ApJ, 371, 684

Bodaghee, A., Walter, R., Zurita Heras, J. A., et al. 2005, A\&A, in press

Bronfman, L., Casassus, S., May, J., \& Nyman, L.-Å. 2000, A\&A, 358, 521

Chevalier, C., \& Ilovaisky, S. A. 1998, A\&A, 330, 201

Corbet, R., Barbier, L., Barthelmy, S., et al. 2005, Am. Astron. Soc. Meet. Abstracts, 207,

Corbet, R. H. D., Hannikainen, D. C., \& Remillard, R. 2004, The Astronomer's Telegram, 269, 1

Courvoisier, T. J.-L., Walter, R., Beckmann, V., et al. 2003, A\&A, 411, L53

Cutri, R. M., Skrutskie, M. F., van Dyk, S., et al. 2003, VizieR Online Data Catalog, 2246, 0

Dickey, J. M., \& Lockman, F. J. 1990, ARA\&A, 28, 215

Filliatre, P., \& Chaty, S. 2004, ApJ, 616, 469

González-Riestra, R., Oosterbroek, T., Kuulkers, E., Orr, A., \& Parmar, A. N. 2004, A\&A, 420, 589

Gros, A., Goldwurm, A., Cadolle-Bel, M., et al. 2003, A\&A, 411, L179

Hickox, R. C., Narayan, R., \& Kallman, T. R. 2004, ApJ, 614, 881

Hill, A., Walter, R., Knigge, C., et al. 2005, A\&A, in press

Hynes, R. I., Clark, J. S., Barsukova, E. A., et al. 2002, A\&A, 392, 991

Illarionov, A. F., \& Sunyaev, R. A. 1975, A\&A, 39, 185

Jansen, F., Lumb, D., Altieri, B., et al. 2001, A\&A, 365, L1
Kuulkers, E. 2005, in Interacting Binaries: Accretion, Evolution, and Outcomes, AIP Conf. Proc., 797, 402

Labanti, C., Di Cocco, G., Ferro, G., et al. 2003, A\&A, 411, L149

Lebrun, F., Leray, J. P., Lavocat, P., et al. 2003, A\&A, 411, L141

Liu, Q. Z., van Paradijs, J., \& van den Heuvel, E. P. J. 2000, A\&AS, 147, 25

Lubinski, P., Chernyakova, M., Kretschmar, P., et al. 2004, ISDC Newsletter, 16

Lund, N., Budtz-Jørgensen, C., Westergaard, N. J., et al. 2003, A\&A, 411, L231

Lutovinov, A., Revnivtsev, M., Molkov, S., \& Sunyaev, R. 2005a, A\&A, 430, 997

Lutovinov, A., Rodriguez, J., Revnivtsev, M., \& Shtykovskiy, P. 2005b, A\&A, 433, L41

Markwardt, C. B., \& Swank, J. H. 2003, The Astronomer's Telegram, 179

Mas-Hesse, J. M., Giménez, A., Culhane, J. L., et al. 2003, A\&A, 411, L261

Matt, G. 2002, MNRAS, 337, 147

Matt, G., \& Guainazzi, M. 2003, MNRAS, 341, L13

Morrison, R., \& McCammon, D. 1983, ApJ, 270, 119

Murakami, H., Dotani, T., \& Wijnands, R. 2003, IAU Circ., 8070, 3

Nagase, F. 2002, Journal of Astrophysics and Astronomy, 23, 59

Negueruela, I. 1998, A\&A, 338, 505

Negueruela, I. 2004, in The Many Scales of the Universe - JENAM 2004 Astrophysics Reviews (Kluwer Academic Publishers) ed. J. C. del Toro Iniesta, et al.

Ochsenbein, F., Bauer, P., \& Marcout, J. 2000, A\&AS, 143, 23

Patel, S. K., Kouveliotou, C., Tennant, A., et al. 2004, ApJ, 602, L45

Predehl, P., \& Schmitt, J. H. M. M. 1995, A\&A, 293, 889

Revnivtsev, M. G., Sazonov, S. Y., Gilfanov, M. R., \& Sunyaev, R. A. 2003, Astron. Lett., 29, 587

Rodriguez, J., Cabanac, C., Hannikainen, D. C., et al. 2005, A\&A, 432, 235

Rodriguez, J., Tomsick, J. A., Foschini, L., et al. 2003, A\&A, 407, L41

Sako, M., Kahn, S., Paerels, F., et al. 2002, in High-resolution X-ray Spectroscopy Workshop with XMM-Newton and Chandra, ed. G. Branduardi-Raymont,

http://www.mssl.ucl.ac.uk/ gbr/rgs_workshop/

Schartel, N., Ehle, M., Breitfellner, M., et al. 2003, IAU Circ., 8072, 3

Schulz, N. S., Canizares, C. R., Lee, J. C., \& Sako, M. 2002, ApJ, 564, L21

Smith, D. M. 2004, The Astronomer's Telegram, 338

Stee, P. 1998, A\&A, 336, 980

Strüder, L., Briel, U., Dennerl, K., et al. 2001, A\&A, 365, L18

Sugizaki, M., Mitsuda, K., Kaneda, H., et al. 2001, ApJS, 134, 77

Turner, M. J. L., Abbey, A., Arnaud, M., et al. 2001, A\&A, 365, L27

Ubertini, P., Lebrun, F., Di Cocco, G., et al. 2003, A\&A, 411, L131

van der Meer, A., Kaper, L., di Salvo, T., Méndez, M., \& van der Klis, M. 2004, in Rev. Mex. Astron. Astrofis. Conf. Ser., 213

van Paradijs, J. 1983, High-mass X-ray Binaries, Cambridge Astrophysics Series (Cambridge, MA: Cambridge University Press)

van Paradijs, J. 1995, in X-ray Binaries, ed. W. H. G. Lewin, J. van Paradijs, E. P. J. van den Heuvel (Cambridge University Press), 536

Vedrenne, G., Roques, J.-P., Schönfelder, V., et al. 2003, A\&A, 411, L63

Walter, R. 2004, in AAS/High Energy Astrophysics Division Meeting 8, 33.01

Walter, R., Courvoisier, R., Foschini, L., et al. 2004, in Proc. of the 5th INTEGRAL workshop, 16-20 February 2004, Munich, Germany, ESA-SP552,417

Walter, R., Favre, P., Dubath, P., et al. 2003a, A\&A, 411, L25

Walter, R., Rodriguez, J., Foschini, L., et al. 2003b, A\&A, 411, L427

Warwick, R. S., Norton, A. J., Turner, M. J. L., Watson, M. G., \& Willingale, R. 1988, MNRAS, 232, 551

Waters, L. B. F. M., \& van Kerkwijk, M. H. 1989, A\&A, 223, 196

Winkler, C., Courvoisier, T. J.-L., Di Cocco, G., et al. 2003, A\&A, 411, L1

Zickgraf, F.-J., Stahl, O., \& Wolf, B. 1992, A\&A, 260, 205

Zurita Heras, J. A., de Cesare, G., Walter, R., et al. 2005, A\&A, in press 